\title{
Combined Gemcitabine and Metronidazole Is a Promising Therapeutic Strategy for Cancer Stem-like Cholangiocarcinoma
}

\author{
MAKOTO KAWAMOTO ${ }^{1,2}$, MASAYO UMEBAYASHI ${ }^{3}$, HIROTO TANAKA ${ }^{3}$, \\ NORIHIRO KOYA ${ }^{3}$, SINICHIRO NAKAGAWA ${ }^{3}$, KEN KAWABE ${ }^{4}$, HIDEYA ONISHI ${ }^{1}$, \\ MASAFUMI NAKAMURA ${ }^{2}$ and TAKASHI MORISAKI ${ }^{3}$ \\ Department of ${ }^{1}$ Cancer Therapy and Research, ${ }^{2}$ Surgery and Oncology, \\ Graduate School of Medical Sciences, Kyushu University, Fukuoka, Japan; \\ ${ }^{3}$ Fukuoka General Cancer Clinic, Fukuoka, Japan; \\ ${ }^{4}$ National Hospital Organization, Kyushu Medical Center, Fukuoka, Japan
}

\begin{abstract}
Background/Aim: Metronidazole (MNZ) is a common antibiotic that exerts disulfiram-like effects when taken together with alcohol. However, the relationship between MNZ and aldehyde dehydrogenase $(A L D H)$ activity remains unclear. This study investigated whether MNZ reduces cancer stemness by suppressing $A L D H$ activity and accordingly reducing the malignancy of cholangiocarcinoma (CCA). Materials and Methods: We developed gemcitabine (GEM)-resistant TFK-1 cells and originally established CCA cell line from a patient with GEM-resistant CCA. Using these cell lines, we analyzed the impacts of MNZ for cancer stem cell markers, invasiveness, and chemosensitivity. Results: MNZ reduced ALDH activity in GEM-resistant CCA cells, leading to decreased invasiveness and enhanced chemosensitivity. MNZ diminished the invasiveness by inducing mesenchymal-epithelial transition and enhancing chemosensitivity by increasing ENT1 (equilibrative nucleoside transporter 1) and reducing RRMI (ribonucleotide reductase M1). Conclusion: MNZ reduced cancer stemness in GEM-resistant CCA cells. Combined GEM and MNZ would be a promising therapeutic strategy for cancer stem-like CAA.
\end{abstract}

Cholangiocarcinoma (CCA) is the most common biliary tract malignancy and second most common primary hepatic malignancy (1). The incidence of CCA is the highest in East

This article is freely accessible online.

Correspondence to: Takashi Morisaki, Fukuoka General Cancer Clinic, 3-1-1 Sumiyoshi, Hakata-ku, Fukuoka 812-0018, Japan. Tel: +81 922827696, Fax: +81924056376, e-mail: tmorisaki@cancerclinic.jp

Key Words: Cholangiocarcinoma, aldehyde dehydrogenase, metronidazole, cancer stem cells, chemoresistance, epithelialmesenchymal transitions. and South Asia and increasing worldwide (2). Curative therapy for CCA is surgical resection, but 5-year survival rates are $25-30 \%$, even for patients who undergo aggressive surgery (3). Unfortunately, non-surgical treatment options such as chemotherapy and radiotherapy are limited and their effects are unsatisfactory. Gemcitabine (GEM) is one of the few drugs for CCA treatment, and resistance to GEM has become a major problem. Therefore, novel therapeutic approaches to enhance GEM sensitivity are greatly and urgently needed.

Cancer stem cells (CSCs), that are critical for tumor initiation, progression, and persistence, have been reported to have increased resistance to treatments such as chemotherapy and radiotherapy (4), and a mesenchymal phenotype (5), leading to recurrence after treatment or metastatic behavior. Therefore, many studies have been carried out to isolate CSCs or reduce cancer stemness features to eradicate CSCs. However, the mechanisms by which chemoresistance and changes in the malignant phenotype of CCA arise are still not elucidated.

Aldehyde dehydrogenase (ALDH) oxidizes aldehydes to carboxylic acids and has been identified as a CSC marker in a number of solid malignancies (6). Additionally, ALDH has been shown to be an indicator of relapse or prognosis in certain cancers $(7,8)$.

Metronidazole (MNZ) is a common oral antibiotic with a high histological migration property and well-established safety. In addition, MNZ exerts disulfiram-like effects when taken together with alcohol (9). Disulfiram has been used for more than six decades in alcohol aversion therapy because of its inhibitory effect on ALDH. Recently, it has attracted significant attention in relation to its anticancer efficacy (10). However, the relationship between MNZ and ALDH remains unclear.

This study tested the hypothesis that MNZ could decrease cancer stemness by inhibiting ALDH activity and accordingly suppressing malignant phenotypes. We derived 
GEM-resistant CCA cells from TFK-1 cells and an originally established CCA cell line from a patient with GEM-resistant CCA, TFK-1 GR and THK-1 cells, respectively. Then, the effects of MNZ on the expression of CSC markers, ALDH activity, and functions of cancer cells were assessed in these cell lines. As a result, we demonstrated for the first time that MNZ decreased the invasive potential and enhanced chemosensitivity to GEM and suppressed ALDH activity in CCA cells. Moreover, we revealed the mechanisms of the changes in invasiveness and chemosensitivity. MNZ diminished the invasive ability by inducing mesenchymalepithelial transition (MET) and enhancing chemosensitivity via increasing equilibrative nucleoside transporter 1 (ENT1) and reducing ribonucleotide reductase M1 (RRM1). These findings provide a new insight into an anticancer strategy to enhance the chemosensitivity of GEM-resistant CCA cells, leading to a better CCA prognosis.

\section{Materials and Methods}

Cell line. TFK-1 cells (11) were purchased from RIKEN BioResource Center (RIKEN BRC, Ibaraki, Japan). The cells were cultured in RPMI 1640 (Nacalai Tesque, Kyoto, Japan) supplemented with 10\% fetal bovine serum (FBS; Life Technologies, Carlsbad, CA, USA), $100 \mathrm{U} / \mathrm{ml}$ penicillin (Meiji Seika Pharma Co., Ltd., Tokyo, Japan), and $100 \mu \mathrm{g} / \mathrm{ml}$ streptomycin (Meiji Seika Pharma Co., Ltd., Tokyo, Japan). There was no mycoplasma contamination as tested by a mycoplasma detection kit (Lonza, Basel, Switzerland).

Establishment and characterization of a CCA cell line from a patient with chemorefractory recurrent $C A A$. Peritoneal fluid was obtained from a 62-year-old male patient with recurrent CCA, who had undergone chemotherapy consisting of GEM and TS-1, and whose disease then progressed to the formation malignant ascites with chemoresistance. Cells in the peritoneal fluid were cultured in Dulbecco's modified Eagle's medium (DMEM)/F12 (Life Technologies, Grand Island, NY, USA) supplemented with $10 \%$ FBS. Written informed consent was obtained from the patient. This study was approved by the Ethical Committee of the Fukuoka General Cancer Clinic.

Aldefluor assay. ALDH activity in viable cells was determined using a fluorogenic dye-based Aldefluor assay (Stem Cell Technologies, Grenoble, France), according to the manufacturer's instructions. A total of $1 \times 10^{6} \mathrm{cells} / \mathrm{ml}$ were suspended in Aldefluor assay buffer containing ALDH substrate (bodipy-aminoacetaldehyde) and incubated for $15 \mathrm{~min}$ at $37^{\circ} \mathrm{C}$. As a reference control, cells were suspended in buffer containing bodipy-aminoacetaldehyde in the presence of diethylaminobenzaldehyde (DEAB), a specific ALDH1 inhibitor. Brightly fluorescent ALDH-expressing cells (ALDH1high) were detected in the green fluorescence channel $(520-540 \mathrm{~nm})$ of the FC500 flow cytometer (Beckman Coulter, Brea, CA, USA).

Cell proliferation assay. Cells were treated with various concentrations of GEM or with MNZ. For proliferation assays, cells were cultured in 96-well plates (Becton Dickinson, Franklin Lakes, $\mathrm{NJ}, \mathrm{USA})$ at $5 \times 10^{4}$ cells $/ \mathrm{ml}$ in $100 \mu \mathrm{l}$ of medium. Assays were performed over $48 \mathrm{~h}$ with a minimum of five replicates.
Proliferation assays were performed using a WST-8 cell proliferation kit (Dojindo, Osaka, Japan), according to the manufacturer's instructions.

Sphere-forming assay. At $80 \%$ confluence, cells were detached using $1 \times$ trypsin-EDTA. The cells were resuspended in serum-free DMEM/F12 containing $20 \mathrm{ng} / \mathrm{ml}$ epidermal growth factor (Invitrogen, Grand Island, NY, USA), and $20 \mathrm{ng} / \mathrm{ml}$ basic fibroblast growth factor (Invitrogen, Seoul, Korea) [DMEG(+)GF], high glucose DMEM with FBS [DMEM(+)FBS], or high glucose DMEM without FBS [DMEM(-)FBS]. Cells were subsequently cultured in ultra-low attachment 96-well plates (Corning, NY, USA) at a density of 2,000 cells per well at $37^{\circ} \mathrm{C}$ with $5 \% \mathrm{CO}_{2}$. After 7 days of culture, sphere numbers were counted in each well.

Cell invasion assay. The invasiveness of TFK-1 and THK-1 cell lines was assessed by a Matrigel invasion assay as described previously (12). The upper surface of a filter (pore size: $8.0 \mu \mathrm{m}$; BD Bioscience, Heidelberg, Germany) was coated with basement membrane Matrigel (BD Bioscience, Heidelberg, Germany). Cells $\left(2 \times 10^{5}\right.$ cells) were placed in the upper chamber with or without $\mathrm{MNZ}$ and subsequently incubated for $24 \mathrm{~h}$. The cells that migrated to the lower side of the filter were fixed, stained with Diff-Quik reagent (Sysmex, Kobe, Japan), and then counted under a light microscope (Eclipse TE 300; Nikon, Tokyo, Japan).

Monoclonal antibodies and flow cytometry. Surface markers of tumor cells were labeled by direct or indirect immunofluorescence using the following monoclonal antibodies: anti-CD44-PE (Immunotech Beckman Coulter, Brea, CA, USA), anti-EpCAM-FITC (BioLegend, San Diego, CA, USA), anti-CD133 (Miltenyi Biotec GmbH, Bergisch Gladbach, Germany), and goat anti-mouse IgG-Alexa Fluor 488 (Molecular Probes, Eugene, OR, USA). To detect CSC-like markers, detached cells were washed twice in cold phosphate-buffered saline and stained with appropriate concentrations of labeled monoclonal antibodies for $1 \mathrm{~h}$ at $4^{\circ} \mathrm{C}$. Fluorescence was detected using the FC500 flow cytometer (Beckman Coulter, Fullerton, CA, USA) and expressed as the relative mean fluorescence intensity or percentage of positive cells.

Calcein-release cytotoxicity assay and cell imaging. Antibodymediated cellular cytotoxicity (ADCC) assays were performed by calcein-AM release. Briefly, target cells were resuspended at $1 \times 10^{5}$ cells $/ \mathrm{ml}$ in complete medium and allowed to adhere in a 96-well plate overnight. Then, the cells were incubated at $37^{\circ} \mathrm{C}$ for $48 \mathrm{~h}$ in the presence or absence of MNZ or GEM at various concentrations. After incubation, the cells were washed with medium, incubated with calcein-AM for $30 \mathrm{~min}$, and then washed again. The plate was washed and the adherent cells were analyzed under a fluorescence microscope (IX81; Olympus, Tokyo, Japan) by Lumina Vision software (version 2.4.2; Mitani, Fukui, Japan). Images were captured with a color CCD camera (DPI72; Olympus) and LUC plan FLN objective lens (Olympus, Tokyo, Japan). All procedures were performed at $20-25^{\circ} \mathrm{C}$.

Western blot analysis. Western blotting was performed as described previously (12). Whole protein extraction was performed with MPER Reagent (Pierce Biotechnology, Rockford, IK, USA), according to the manufacturer's instructions. Protein concentrations were determined with the Bio-Rad Protein Assay (Bio-Rad, 
Hercules, CA, USA). The whole protein extract $(50 \mu \mathrm{g})$ was separated by electrophoresis on a polyacrylamide gel and transferred to polyvinylidene fluoride membranes (Bio-Rad, Hercules, CA, USA). Membranes were incubated overnight at $4^{\circ} \mathrm{C}$ with primary antibodies against E-cadherin (1:200, sc-7870; Santa Cruz Biotechnology, Santa Cruz, CA, USA), vimentin (1:200, sc-6260; Santa Cruz Biotechnology, Santa Cruz, CA, USA), Slug (1:200, sc15391; Santa Cruz Biotechnology, Santa Cruz, CA, USA), Twist (1:200, sc-15393; Santa Cruz Biotechnology, Santa Cruz, CA, USA), ENT1 (1:200, sc-377283; Santa Cruz Biotechnology, Santa Cruz, CA, USA), and RRM1 (1:10,000, ab137114; Abcam, Cambridge, MA, USA). Peroxidase-linked secondary antibodies (Amersham Biosciences, Piscataway, NJ, USA) were subsequently added, and the membranes were further incubated for $1 \mathrm{~h}$ at room temperature. An antibody against $\alpha$-tubulin $(1: 1,000$, SigmaAldrich, St. Louis, MO, USA) was used for protein loading controls.

Statistical analysis. All data are represented as the mean \pm standard deviation (SD). The Student's $t$-test was used for comparison of mean values between two groups. A value of $p<0.05$ was considered significant.

\section{Results}

GEM-resistant TFK-1 (TFK-1 GR) cells exhibit higher CSC marker expression and $A L D H$ activity. One of the hallmarks of CSCs is their resistance to conventional chemotherapeutic agents. We hypothesized that cancer stemness features are enhanced in GEM-resistant CCA cells. To test this hypothesis, we first derived a stable CCA cell line that was chronically resistant to GEM using TFK-1 cells. Specifically, TFK-1 cells in the logarithmic growth phase were first exposed to 0.001 $\mu \mathrm{g} / \mathrm{ml}$ GEM, which resulted in greater than $95 \%$ cell death. Once surviving cells reached $80 \%$ confluence, they were passaged twice in the same concentration of GEM. The process was repeated with increasing doses of GEM until a cell population was selected, which demonstrated resistance to at least $0.2 \mu \mathrm{g} / \mathrm{ml}$ GEM. This cell line was designated as TFK-1 GR. The GEM-resistant phenotype has been stable for more than 6 months of passaging to date. Cancer stemness features represented by cell surface markers or ALDH activity were compared between parental TFK-1 and TFK-1 GR cells.

As shown in Figure 1A, TFK-1 GR cells were confirmed to have acquired GEM resistance compared with parental TFK-1 cells. CSC surface markers (CD44, CD133, and EpCAM) and CSC functional marker ALDH were assessed by flow cytometry. The mean fluorescence intensities of CD44, CD133, and EpCAM were higher in TFK-1 GR cells than parental TFK-1 cells (Figure 1B). Furthermore, the mean frequencies and mean fluorescence intensities of ALDH activity were enhanced in TFK-1 GR cells (Figure 1C). These results suggest that GEM-resistant CCA cells had acquired cancer stemness features during GEM treatment.

MNZ decreases ALDH activity in TFK-1 GR cells. Next, we determined whether MNZ inhibited ALDH activity, a commonly used CSC functional marker, in TFK-1 GR cells . MNZ alone did not show any cytotoxicity in both cell lines (Figure 1A). As shown in Figure 2A, treatment with MNZ significantly decreased ALDH activity in TFK-1 GR cells. Additionally, sphere-forming assays were conducted to assess self-renewal capacity, another functional CSC characteristic. An enhanced sphere-forming ability in TFK1 GR cells was abrogated by MNZ treatment in a dose dependent manner (Figure 2B). These results suggest that MNZ may be useful to suppress cancer stemness features of CCA cells with GEM resistance.

MNZ decreases the invasiveness of GEM-resistant TFK-1 cells. A previous report has shown that silencing ALDH1A3, an isoform of the ALDH superfamily, contributes to MET in CCA (8). A characteristic of mesenchymal cells is an increased invasive potential. This finding encouraged us to hypothesize that MNZ could inhibit the invasive potential of CCA cells by decreasing ALDH activity. To investigate the effects of MNZ on the invasiveness of CCA cells, Matrigel invasion assays were performed using parental TFK-1 and TFK-1 GR cells. We first confirmed that TFK-1 GR cells exhibited a higher invasive potential than parental TFK-1 cells (Figure 3A). Interestingly, MNZ treatment significantly suppressed the invasive potential of TFK-1 GR cells in a dose dependent manner (Figure 3B).

These results suggest that MNZ inhibits invasiveness by decreasing ALDH activity in GEM-resistant CCA cells.

MNZ diminishes the invasive ability of TFK-1 GR cells by inducing MET. We examined whether altering the expression levels of ALDH by MNZ in TFK-1 GR cells affected their epithelial-mesenchymal transition (EMT) phenotype. We first observed morphological changes in phase contrast images. We found that TFK-1 GR cells showed a scattered phenotype compared with TFK-1 cells, suggesting that TFK-1 GR cells lost cell-cell contacts and acquired motility, which is one of the major characteristics of EMT. In contrast, TFK-1 GR cells treated with MNZ showed a clustering phenotype, suggesting that MNZ abrogated EMT (Figure 4A). Second, to determine whether these morphological transformations represented EMT, we analyzed the expression of E-cadherin, vimentin, and EMT transcription factors (Slug and Twist) by western blotting. As expected, MNZ exposure up-regulated the expression of E-cadherin and decreased expression of vimentin and EMT transcription factors (Figure 4B).

Taken together, these results suggested that MNZ reduced ALDH, leading to MET of TFK-1 GR cells and a diminished invasive ability.

MNZ enhances GEM sensitivity of TFK-1 GR cells by reducing $R R M 1$ and increasing ENT1. Previous studies have reported that $\mathrm{ALDH}^{\text {high }}$ cells or mesenchymal cells exhibit chemoresistance. 

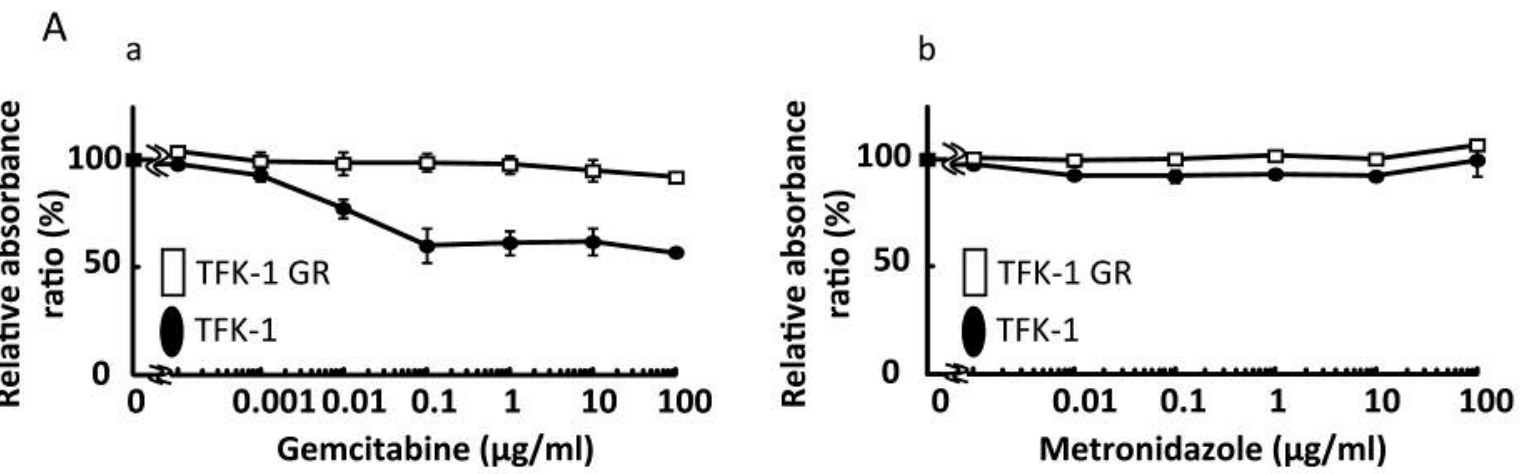

B

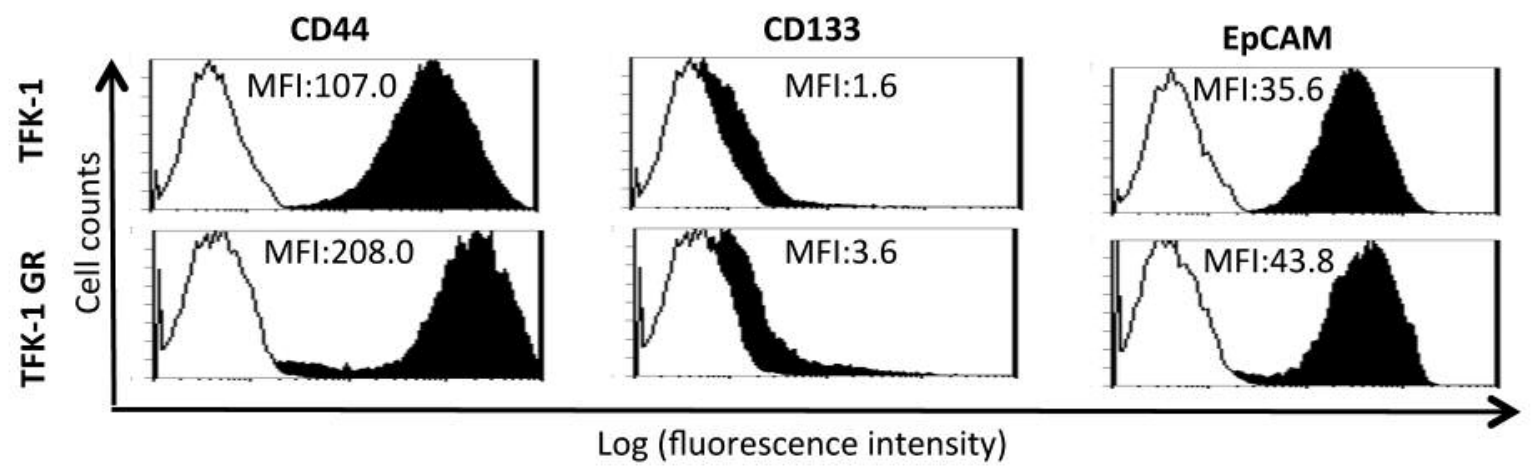

C
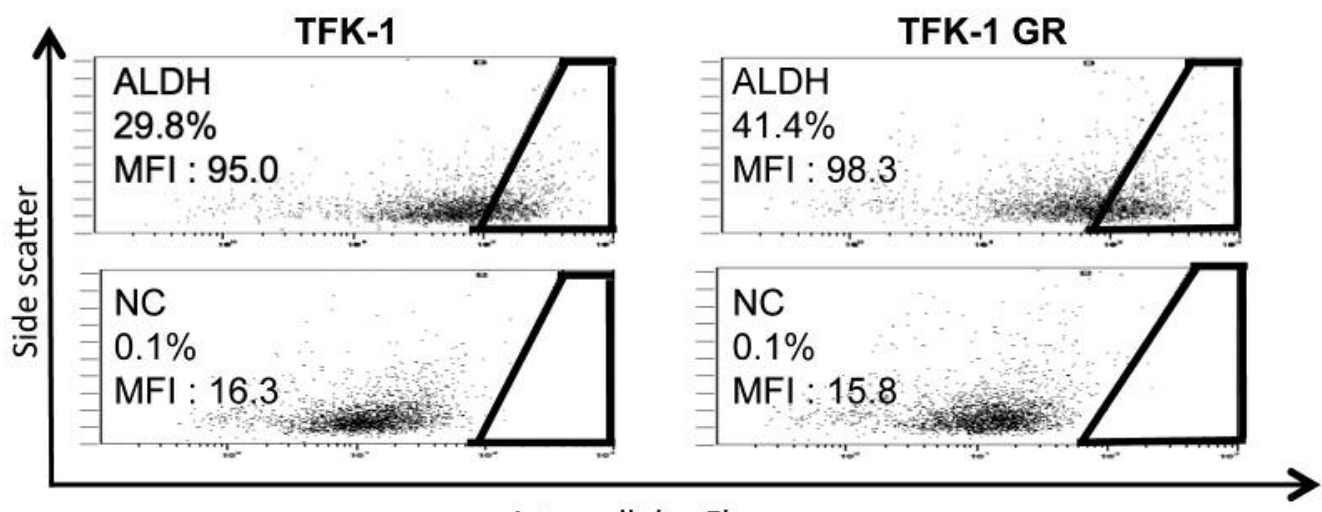

Intracellular-Fluorescence

Figure 1. GEM-resistant CCA cells exhibit enhanced CSC surface markers and ALDH activity. (A) Growth inhibitory effects on TFK-1 and TFK-1 GR cells. Cells were treated with GEM or MNZ at 0-100 $\mu \mathrm{g} / \mathrm{ml}$ for $48 \mathrm{~h}$, and growth inhibition was quantified by the WST-8 assay. (a) Representative data of growth inhibitory effects of GEM. (b) Representative data of growth inhibitory effects of MNZ. (B) CSC surface marker expression was analyzed by flow cytometry in TFK-1 and TFK-1 GR cells. Representative histograms show CD44/CD133/EpCAM expression (black profiles) relative to the IgG isotype control (white profiles). (C) ALDH activity in TFK-1 and TFK-1 GR cells. The percentages of ALDH ${ }^{+}$cells were assessed using the Aldefluor assay. Cells in the trapezoid region were considered to express ALDH. MFI: Mean fluorescence intensity.

We then analyzed whether MNZ-induced ALDH suppression affects GEM sensitivity in TFK-1 GR cells. As shown in Figure $5 \mathrm{~A}, \mathrm{MNZ}$ exposure did not result in a significant difference in the proliferative potential. However, MNZ treatment enhanced chemosensitivity to GEM in TFK-1 GR cells in a dose dependent manner, but not in TFK-1 cells (Figure 5B). We assumed that MNZ treatment had no effect on GEM sensitivity in TFK-1 cells because TFK-1 cells had a smaller ALDHpositive population than TFK-1 GR cells. To investigate the mechanism of changes in chemosensitivity, we analyzed the 
A

\section{TFK-1 GR}

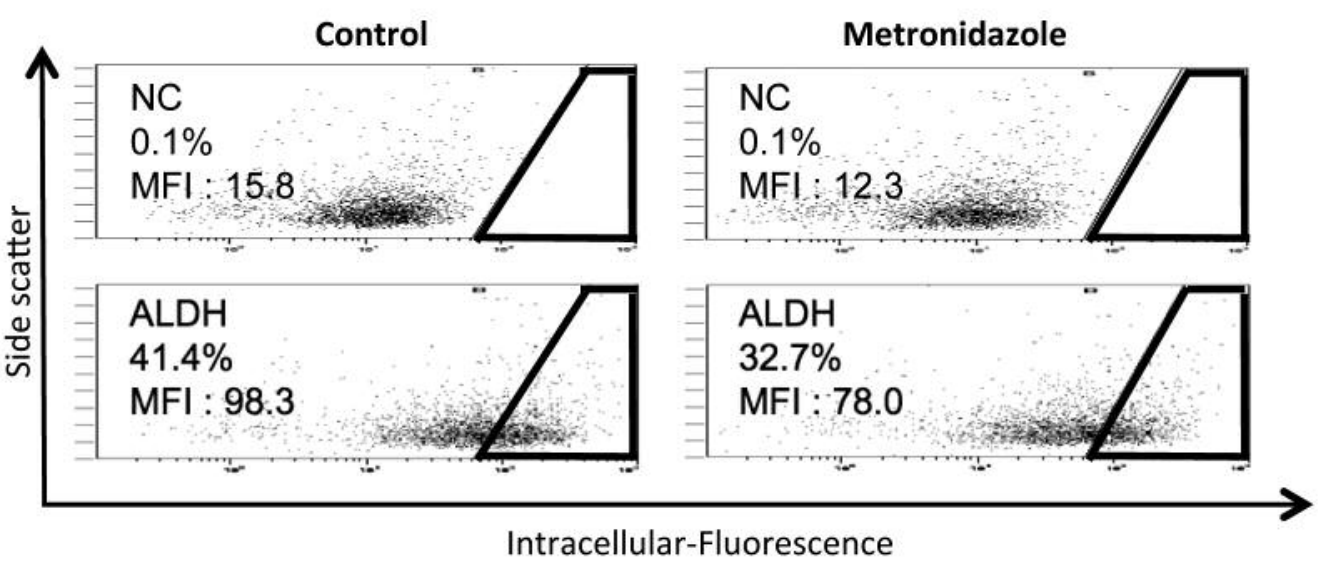

B

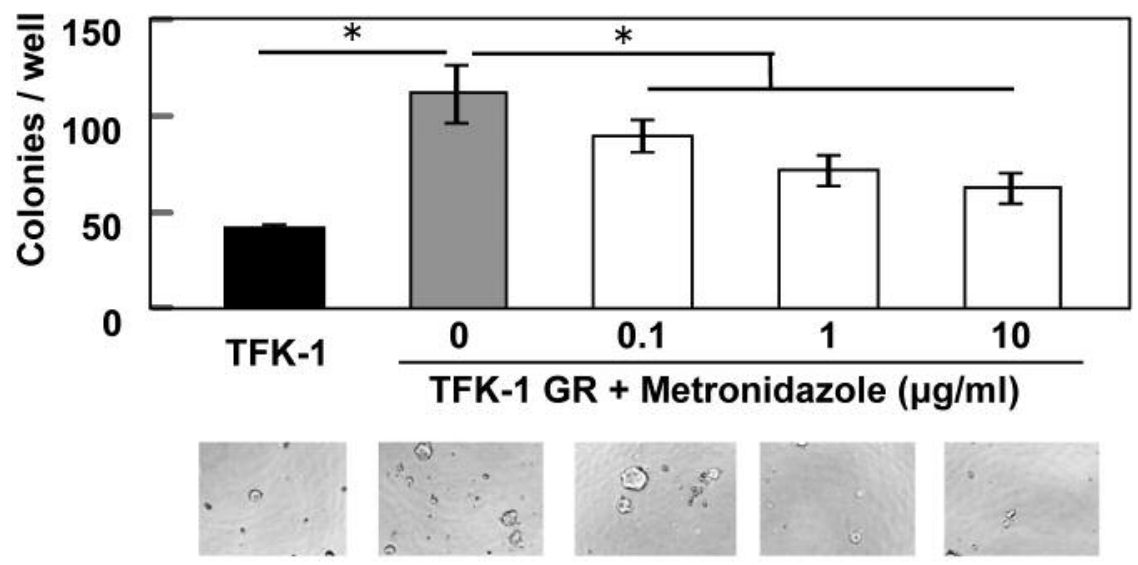

Figure 2. MNZ decreases ALDH activity in TFK-1 GR cells. (A) ALDH activity in TFK-1 GR cells with or without MNZ (0.1 $\mu \mathrm{g} / \mathrm{ml})$ treatment for $48 \mathrm{~h}$. The percentages of $\mathrm{ALDH} \mathrm{H}^{+}$cells were assessed using the Aldefluor assay. MFI: Mean fluorescence intensity. (B) Sphere-forming assay of TFK-1 and TFK-1 GR cells. TFK-1 GR cells were treated with 0-10 $\mu \mathrm{g} / \mathrm{ml}$ MNZ for 7 days, and then spheres were counted in each well. Representative images are shown. Original magnification: $\times 100 . * p<0.05$. Error bars represent $S D$.

expression of RRM1 and ENT1. MNZ treatment decreased the expression of RRM1 and increased ENT1 expression in TFK-1 GR cells treated with MNZ, which might explain the increased sensitivity of TFK-1 GR cells to GEM (Figure 4B). These results suggested that MNZ-induced down-regulation of ALDH decreased RRM1 and increased ENT1, resulting in enhanced chemosensitivity to GEM in TFK-1 GR cells.

MNZ decreases ALDH activity and enhances GEM sensitivity of the CCA cell line established from a patient with GEMresistant $C C A$. To confirm our results in vitro, we established a CCA cell line from a patient with GEM-resistant CCA, that was named THK-1. Specifically, the patient received GEMbased chemotherapy for postoperative recurrence and then became resistant to treatment with ascites. THK-1 cells were established from these ascites. CSC surface markers (CD44,
CD133, and EpCAM), ALDH activity, and chemosensitivity to GEM are shown in Figure 6A. MNZ did not significantly affect the viability of THK-1 cells (Figure 6B). Consistent with the aforementioned in vitro results, MNZ treatment decreased ALDH activity (Figure 6C) and enhanced chemosensitivity to GEM in THK-1 cells in a dose-dependent manner (Figure 6D).

Taken together, these results suggest more strongly that MNZ reduces cancer stemness by reducing ALDH activity, resulting in reduction of malignant phenotypes in patients with GEM-resistant CCA.

\section{Discussion}

CSCs, which are critical for tumor initiation, progression and persistence, are considered to be resistant to conventional chemotherapy $(13,14)$. Additionally, many studies have shown 
A

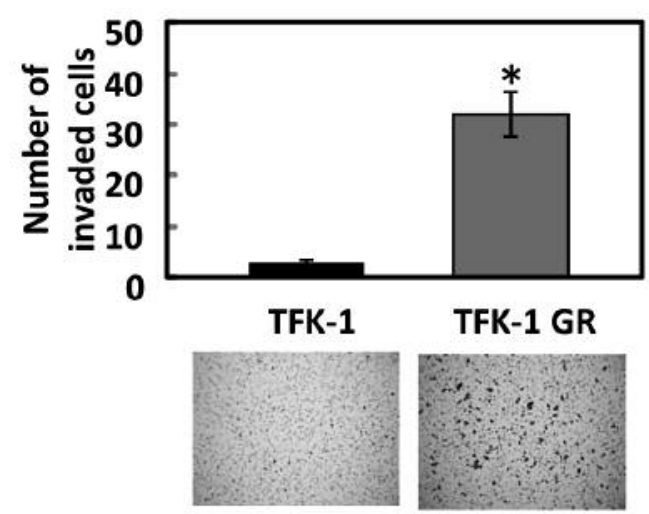

B

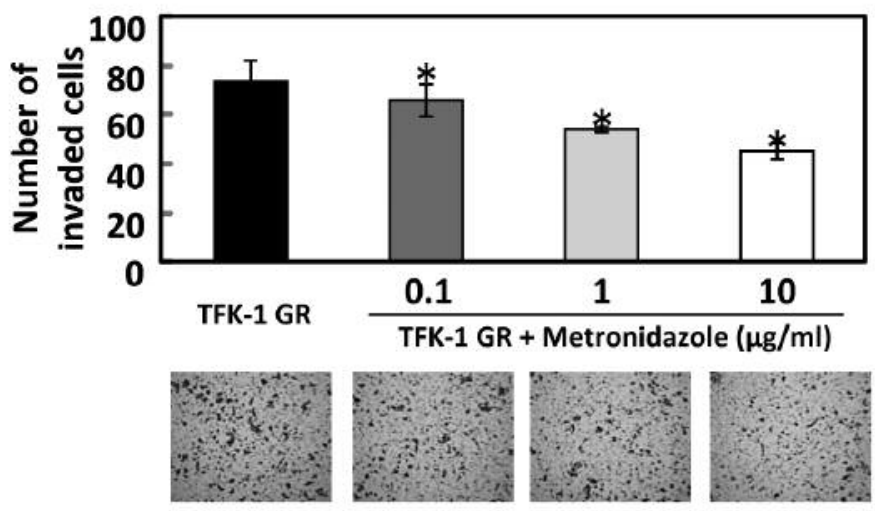

Figure 3. MNZ decreases invasiveness of GEM-resistant TFK-1 cells. (A) Invasion assay of TFK-1 and TFK-1 GR cells. Cells were added to the upper surface of a transmembrane coated with Matrigel. After $24 \mathrm{~h}$ of incubation, total cells that had migrated to the lower surface of the transmembrane were counted. Representative images are shown. Original magnification: $\times 40 . * p<0.05$. Error bars represent SD. (B) Invasion assay of TFK-1 GR cells. Cells were added to the upper surface of a transmembrane coated with Matrigel and treated with 0-10 $\mu$ g/ml MNZ. After 24 h of incubation, total cells that had migrated to the lower surface of the transmembrane were counted. Representative images are shown. Original magnification: $\times 40 . * p<0.05$. Error bars represent $S D$.

A

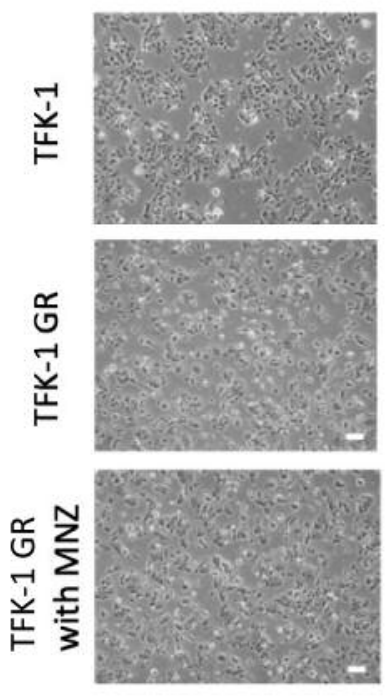

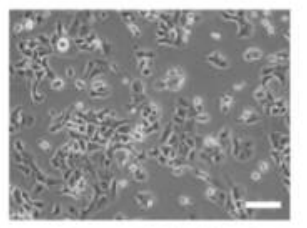
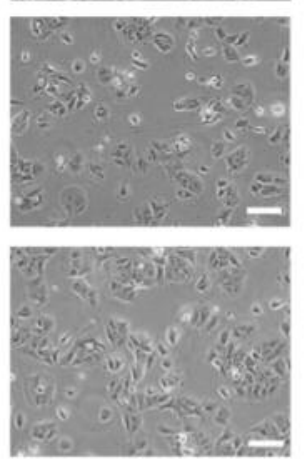

B

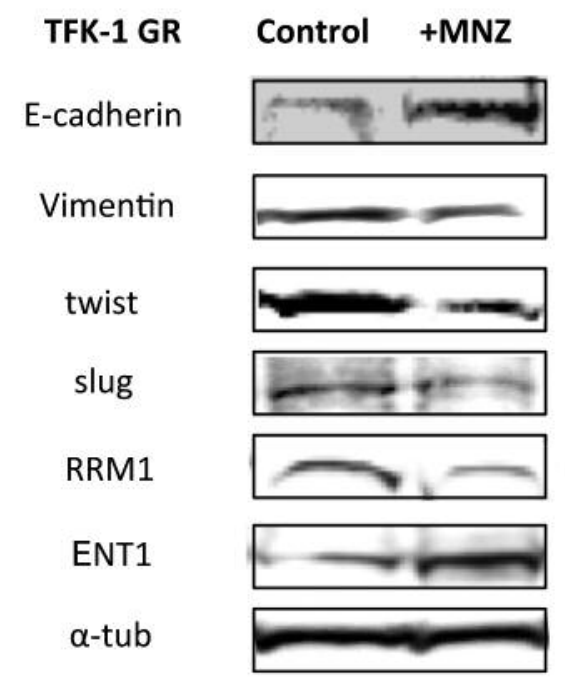

Figure 4. MNZ diminishes the invasive ability of TFK-1 GR cells by inducing MET. (A) Representative cellular morphological images of TFK-1, TFK-1 GR, and TFK-1 GR cells treated with MNZ (100 $\mathrm{g} / \mathrm{ml})$. Original magnifications: $\times 40$ (left) and $\times 100$ (right). Scale bar: $100 \mu \mathrm{m}$. $(B)$ Western blot analysis of E-cadherin, vimentin, and EMT-related transcription factors (Slug and Twist) in TFK-1 GR cells with or without MNZ treatment. Western blot analysis of RRM1 and ENT1 in TFK-1 GR cells with or without MNZ (100 $\mu \mathrm{g} / \mathrm{ml})$ treatment.

that CSCs possess characteristics of mesenchymal cells, contributing to tumor metastasis (15). Therefore, eradication of CSCs is essential to inhibit tumor recurrence and metastasis.

In the past few years, isolation and characterization of stem cells have been attempted for several human tumors. Markers of CSCs can be classified into surface markers (e.g. CD24, CD44, CD133, and EpCAM) and functional markers (e.g. ALDH and SP). CD24, CD44, and EpCAM individually or in combination are markers for CSCs in breast (16), pancreas (17), and colorectal cancers (18). Additionally, CSC surface 
A

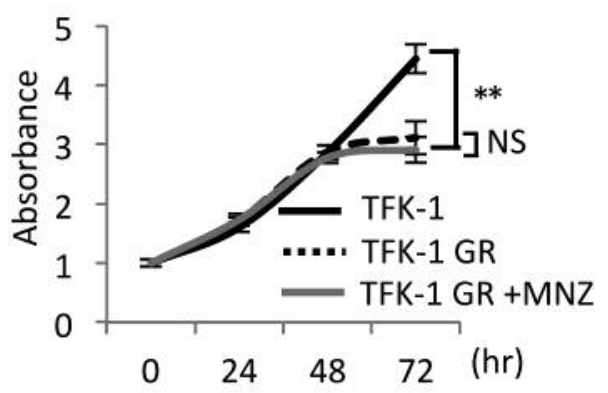

B

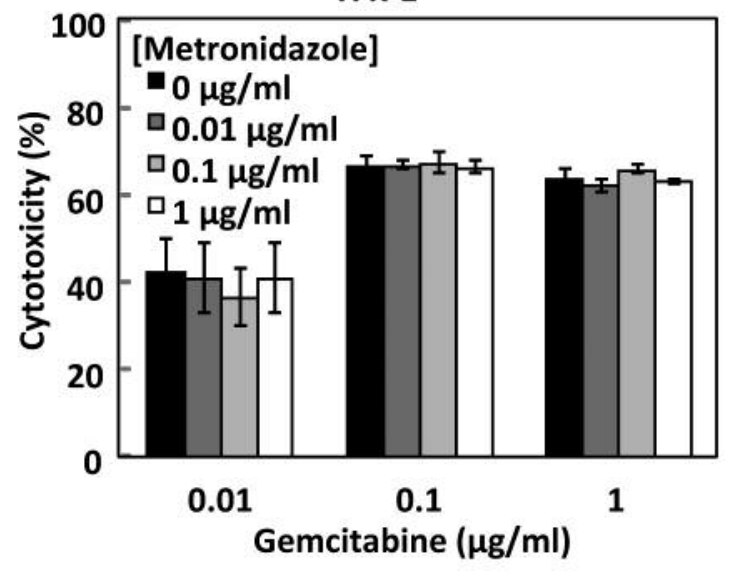

TFK-1 GR

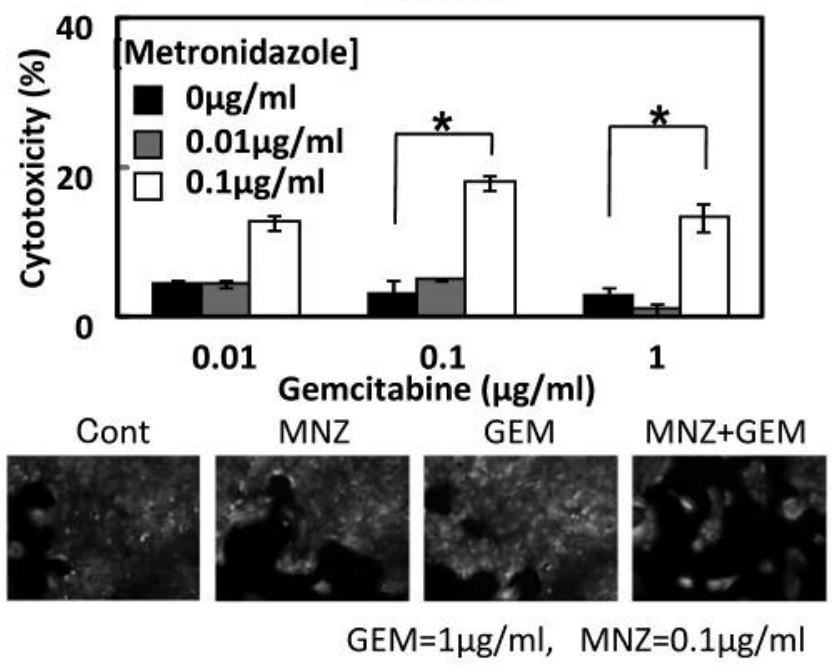

Figure 5. MNZ enhances GEM sensitivity of TFK-1 GR cells by reducing RRM1 and increasing ENT1. (A) Proliferation assay of TFK-1 and TFK$1 \mathrm{GR}$ cells treated with or without MNZ for 24,48 , or $72 \mathrm{~h}$. Cell proliferation was assessed by absorbance. *p<0.05. Error bars represent SD. (B) Synergistic effect of GEM $(0.01-1 \mu \mathrm{g} / \mathrm{ml})$ and MNZ $(0-0.1 \mu \mathrm{g} / \mathrm{ml})$ used in combination against TFK-1 or TFK-1 GR cells. Cytotoxic effects of GEM were assessed by calcein fluorescence intensity at $72 \mathrm{~h}$ post-labeling (top). Cell cultures were loaded with calcein-AM, and fluorescence microscopy $(\times 10)$ was used to detect changes in intracellular calcein fluorescence over time (bottom). Original magnification: $\times 200 . * p<0.05$. Error bars represent $S D$.

markers have been found to be independent prognostic factors for various cancers $(19,20)$. The first study reporting the identification of CCA cells with CSC properties examined the expression of CD24, CD44, and EpCAM (21). A recent study has demonstrated that high expression of CD133 is significantly correlated with poor prognoses of CCA patients (19). Based on these previous studies, we examined the expression of the markers CD44, CD133, and EpCAM.

In humans, the ALDH superfamily of enzymes is responsible for converting both exogenous and endogeneous aldehydes to carboxylic acids (22). Elevated ALDH activity is observed in a variety of cancers, although which type of ALDH isoform regulates ALDH activity differs depending on the type of cancer. ALDH1 serves as a marker of CSCs in a wide range of solid tumors such as head and neck (23), pancreas (17), lung (24), bladder (25), ovary (26), breast (27), and colon (28). ALDH1A3 has been reported to affect prognosis and GEM resistance of CCA (8).

Disulfiram has been shown to be effective against diverse cancer types in preclinical studies. Inhibition of ALDH by disulfiram is supposed to be one of the mechanisms of its anticancer effects exerted by disulfiram (29) No reports have investigated the relationships between $\mathrm{MNZ}$ and the inhibitory effect on ALDH or anticancer effects. We hypothesized that MNZ, which has disulfiram-like effects when taken together with alcohol, could enhance chemosensitivity by inhibiting ALDH activity and consequently reducing recurrence after treatment. 
A
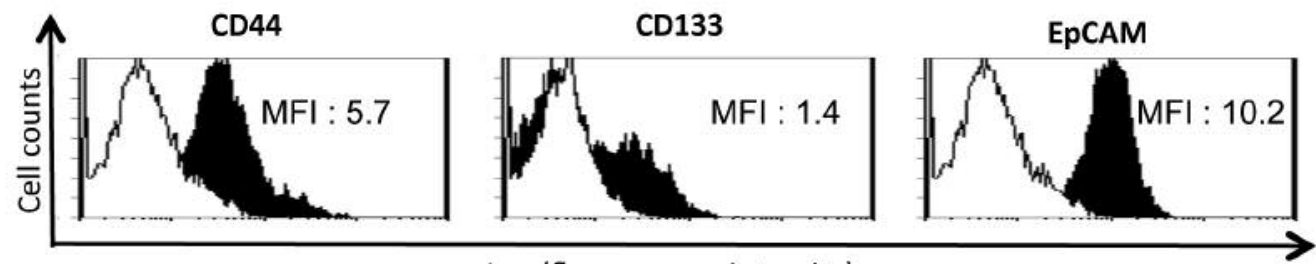

B

Log (fluorescence intensity)
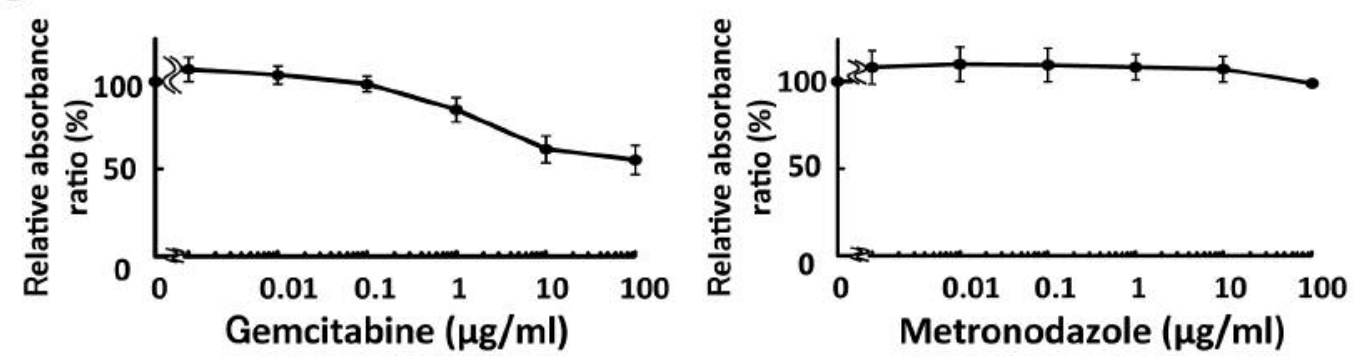

C

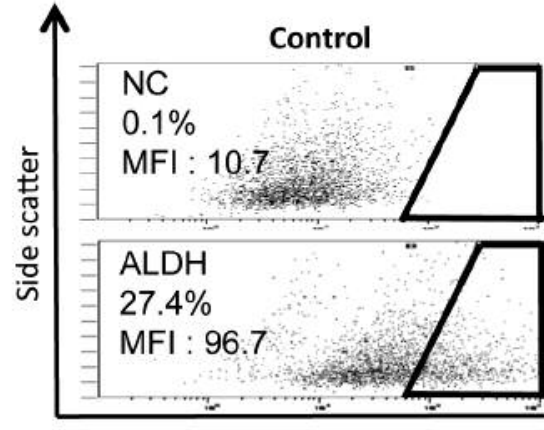

THK-1

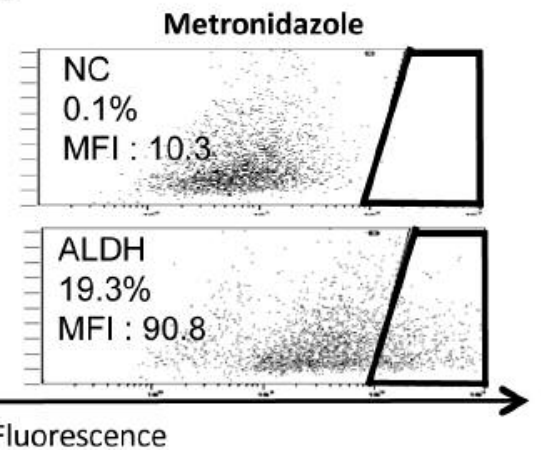

D

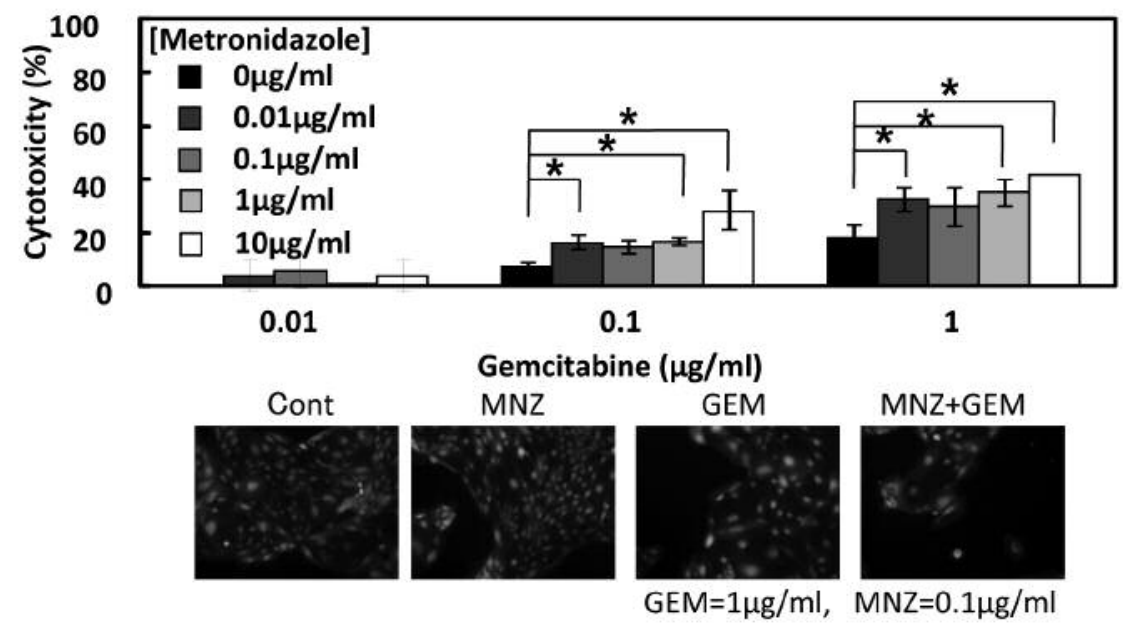

Figure 6. MNZ decreases ALDH activity and enhances GEM sensitivity of the CCA cell line established from a patient with GEM-resistant CCA. (A) CSC surface marker expression and ALDH activity profiles in THK-1 cells. Representative histograms of CD44/CD133/EpCAM expression (black profiles) relative to the IgG isotype control (white profiles) are shown. The percentages of $A L D H^{+}$cells were assessed using the Aldefluor assay. MFI: Mean fluorescence intensity. (B) Growth inhibitory effects on THK-1 cells. Cells were treated with GEM or MNZ at 0-100 $\mu \mathrm{g} / \mathrm{ml}$ for 48 h, and growth inhibition was quantified by the WST-8 assay. Left panel: Representative data of growth inhibitory effects of GEM. Right panel: Representative data of growth inhibitory effects of MNZ. (C) ALDH activity in THK-1 cells treated with or without MNZ (0.1 $\mu \mathrm{g} / \mathrm{ml})$. The percentages of $\mathrm{ALDH}^{+}$cells were assessed using the Aldefluor assay. Cells in the trapezoid region were considered to express ALDH. (D) Synergistic effect of GEM $(0.01-1 \mu \mathrm{g} / \mathrm{ml})$ and $M N Z(0-10 \mu \mathrm{g} / \mathrm{ml})$ used in combination against THK-1 cells. Cytotoxic effects were assessed by calcein fluorescence intensity at 72 h post-labeling (top). Cell cultures were loaded with calcein-AM, and fluorescence microscopy $(\times 10)$ was used to detect changes in intracellular calcein fluorescence over time (bottom). Original magnification: $\times 200 . * p<0.05$. Error bars represent SD. 
Mounting evidence has indicated many features that overlap between CSCs and mesenchymal cells generated by EMT (5). Specifically, recent studies have shown that signaling pathways such as TGF- $\beta 1$, Hedgehog, and Notch not only increase the metastatic potential by EMT, but also contribute to recurrence and drug resistance through the acquisition of stemness under certain conditions (30-32). Our data showing that MNZ reduced ALDH activity and inhibited invasiveness and chemoresistance further support a close relationship between CSCs and mesenchymal cells generated by EMT. CSCs are slow-growing cells that are generally quiescent and therefore resistant to drugs that target fast growing cancer cells (33). Despite our expectations, MNZ exposure did not result in a significant difference in proliferative activity in our experiments. Activities of ENT1 and RRM1 are considered to be key determinants of GEM cytotoxicity (34). ENT1 is a membrane transporter protein that controls efficient permeation of GEM into cells (35). RRM1 is a multimeric enzyme that converts ribonucleotides to deoxyribonucleosides, which is a crucial step for DNA polymerization and repair (36). Previous studies have suggested that ENT, dCK, and RRM1 levels can predict the efficacy of GEM and the prognosis of pancreatic cancer patients (34). To our surprise, MNZ exposure enhanced GEM sensitivity by increasing ENT1 and reducing RRM1 without causing any significant difference in their proliferative activity.

Considering the high costs and long periods of developing new medications, using drugs that are approved for the treatment of diverse diseases as candidate anti-cancer therapeutics is ideal because they benefit from suitable clinically available formulations and evidence of tolerability in patients. MNZ is a common antibiotic with wellestablished pharmacokinetics, safety and tolerance. Importantly, it should be noted that the drug concentration used in this study is much lower than that for treatment of infectious diseases. Additionally, potential advantages of MNZ include its effects on a broad spectrum of malignancies because ALDH activity is a hallmark of CSCs in various cancers. Based on these viewpoints, clinical trials are strongly desired.

There are some limitations in our study such as unassessed ALDH isoforms, only two cell lines were used, and only in vitro experiments were performed.

Collectively, we demonstrated, for the first time, that $\mathrm{MNZ}$ reduces cancer stemness by reducing ALDH activity in CCA cells, leading to a decreased invasive potential and enhanced GEM sensitivity. Additionally, we revealed the mechanisms of the changes in invasiveness and GEM sensitivity. We believe that such a unique combinatorial therapy is quite feasible and may contribute to eradication of GEM-resistant CCA cells and therefore has the potential to improve the prognosis of CCA patients.

\section{Conflicts of Interest}

The Authors declare no potential conflicts of interest.

\section{Acknowledgments}

The Authors thank M. Arico from Edanz Group (www.edanzediting.com/ac) for editing a draft of this manuscript. This study was supported by JSPS KAKENHI Grant Number $17 \mathrm{H} 04283$ and 16K10603.

\section{References}

1 Everhart JE and Ruhl CE: Burden of digestive diseases in the united states part III: Liver, biliary tract, and pancreas. Gastroenterology 136: 1134-1144, 2009.

2 Hughes T, O'Connor T, Techasen A, Namwat N, Loilome W, Andrews RH, Khuntikeo N, Yongvanit P, Sithithaworn P and Taylor-Robinson SD: Opisthorchiasis and cholangiocarcinoma in southeast asia: An unresolved problem. Int J Gen Med 10: 227-237, 2017.

3 Cidon EU: Resectable cholangiocarcinoma: Reviewing the role of adjuvant strategies. Clin Med Insights Oncol 10: 43-48, 2016.

4 Frank NY, Schatton T and Frank MH: The therapeutic promise of the cancer stem cell concept. J Clin Invest 120: 41-50, 2010 .

5 van der Pluijm G: Epithelial plasticity, cancer stem cells and bone metastasis formation. Bone 48: 37-43, 2011.

6 Clark DW and Palle K: Aldehyde dehydrogenases in cancer stem cells: Potential as therapeutic targets. Ann Transl Med 4: 518, 2016.

7 Deng Y, Zhou J, Fang L, Cai Y, Ke J, Xie X, Huang Y, Huang $\mathrm{M}$ and Wang J: ALDH1 is an independent prognostic factor for patients with stages II-III rectal cancer after receiving radiochemotherapy. Br J Cancer 110: 430-434, 2014.

8 Chen MH, Weng JJ, Cheng CT, Wu RC, Huang SC, Wu CE, Chung YH, Liu CY, Chang MH, Chen MH, Chiang KC, Yeh TS, $\mathrm{Su} \mathrm{Y}$ and Yeh CN: ALDH1A3, the major aldehyde dehydrogenase isoform in human cholangiocarcinoma cells, affects prognosis and gemcitabine resistance in cholangiocarcinoma patients. Clin Cancer Res 22: 4225-4235, 2016

9 Finegold SM: Metronidazole. Ann Intern Med 93: 585-587, 1980.

10 Skrott Z, Mistrik M, Andersen KK, Friis S, Majera D, Gursky J, Ozdian T, Bartkova J, Turi Z, Moudry P, Kraus M, Michalova M, Vaclavkova J, Dzubak P, Vrobel I, Pouckova P, Sedlacek J, Miklovicova A, Kutt A, Li J, Mattova J, Driessen C, Dou QP, Olsen J, Hajduch M, Cvek B, Deshales RJ and Bartek J: Alcohol-abuse drug disulfiram targets cancer via p97 segregase adaptor NPL4. Nature 552: 194-199, 2017.

11 Saijyo S, Kudo T, Suzuki M, Katayose Y, Shinoda M, Muto T, Fukuhara K, Suzuki T and Matsuno S: Establishment of a new extrahepatic bile duct carcinoma cell line, TFK-1. Tohoku J Exp Med 177: 61-71, 1995.

12 Kawamoto M, Onishi H, Ozono K, Yamasaki A, Imaizumi A, Kamakura S, Nakano K, Oda Y, Sumimoto H and Nakamura M: Tropomyosin-related kinase B mediated signaling contributes to the induction of malignant phenotype of gallbladder cancer. Oncotarget 8: 36211-36224, 2017. 
13 Nishikawa S, Konno M, Hamabe A, Hasegawa S, Kano Y, Ohta K, Fukusumi T, Sakai D, Kudo T, Haraguchi N, Satoh T, Takiguchi S, Mori M, Doki $\mathrm{Y}$ and Ishii $\mathrm{H}$ : Aldehyde dehydrogenase high gastric cancer stem cells are resistant to chemotherapy. Int J Oncol 42: 1437-1442, 2013.

14 Govaere O, Wouters J, Petz M, Vandewynckel YP, Van den Eynde K, Van den Broeck A, Verhulst S, Dolle L, Gremeaux L, Ceulemans A, Nevens F, van Grunsven LA, Topal B, Vankelecom H, Giannelli G, Van Vlierberghe H, Mikulits W, Komuta $\mathrm{M}$ and Roskams T: Laminin-332 sustains chemoresistance and quiescence as part of the human hepatic cancer stem cell niche. J Hepatol 64: 609-617, 2016.

15 Shuang ZY, Wu WC, Xu J, Lin G, Liu YC, Lao XM, Zheng L and Li S: Transforming growth factor-beta1-induced epithelialmesenchymal transition generates ALDH-positive cells with stem cell properties in cholangiocarcinoma. Cancer Lett 354: 320-328, 2014.

16 Croker AK, Goodale D, Chu J, Postenka C, Hedley BD, Hess DA and Allan AL: High aldehyde dehydrogenase and expression of cancer stem cell markers selects for breast cancer cells with enhanced malignant and metastatic ability. J Cell Mol Med 13: 2236-2252, 2009.

17 Rao CV and Mohammed A: New insights into pancreatic cancer stem cells. World J Stem Cells 7: 547-555, 2015.

18 Huang R, Wang G, Song Y, Tang Q, You Q, Liu Z, Chen Y, Zhang Q, Li J, Muhammand S and Wang X: Colorectal cancer stem cell and chemoresistant colorectal cancer cell phenotypes and increased sensitivity to notch pathway inhibitor. Mol Med Rep 12: 2417-2424, 2015.

19 Fan L, He F, Liu H, Zhu J, Liu Y, Yin Z, Wang L, Guo Y, Wang Z, Yan Q and Huang G: CD133: A potential indicator for differentiation and prognosis of human cholangiocarcinoma. BMC Cancer 11: 320, 2011.

20 Horimoto Y, Arakawa A, Sasahara N, Tanabe M, Sai S, Himuro $\mathrm{T}$ and Saito M: Combination of cancer stem cell markers CD44 and CD24 is superior to ALDH1 as a prognostic indicator in breast cancer patients with distant metastases. PLoS One 11: e0165253, 2016.

21 Wang M, Xiao J, Shen M, Yahong Y, Tian R, Zhu F, Jiang J, Du $\mathrm{Z}, \mathrm{Hu} \mathrm{J}, \mathrm{Liu} \mathrm{W}$ and Qin R: Isolation and characterization of tumorigenic extrahepatic cholangiocarcinoma cells with stem cell-like properties. Int J Cancer 128: 72-81, 2011.

22 Vasiliou V, Pappa A and Petersen DR: Role of aldehyde dehydrogenases in endogenous and xenobiotic metabolism. Chem Biol Interact 129: 1-19, 2000.

23 Chen YC, Chen YW, Hsu HS, Tseng LM, Huang PI, Lu KH, Chen DT, Tai LK, Yung MC, Chang SC, Ku HH, Chiou DH and Lo WL: Aldehyde dehydrogenase 1 is a putative marker for cancer stem cells in head and neck squamous cancer. Biochem Biophys Res Commun 385: 307-313, 2009.

24 Jiang F, Qiu Q, Khanna A, Todd NW, Deepak J, Xing L, Wang H, Liu Z, Su Y, Stass SA and Katz RL: Aldehyde dehydrogenase 1 is a tumor stem cell-associated marker in lung cancer. Mol Cancer Res 7: 330-338, 2009.

25 Keymoosi H, Gheytanchi E, Asgari M, Shariftabrizi A and Madjd Z: ALDH1 in combination with CD44 as putative cancer stem cell markers are correlated with poor prognosis in urothelial carcinoma of the urinary bladder. Asian Pac J Cancer Prev 15: 2013-2020, 2014.
26 Wang YC, Yo YT, Lee HY, Liao YP, Chao TK, Su PH and Lai HC: ALDH1-bright epithelial ovarian cancer cells are associated with CD44 expression, drug resistance, and poor clinical outcome. Am J Pathol 180: 1159-1169, 2012.

27 Morimoto K, Kim SJ, Tanei T, Shimazu K, Tanji Y, Taguchi T, Tamaki Y, Terada N and Noguchi S: Stem cell marker aldehyde dehydrogenase 1-positive breast cancers are characterized by negative estrogen receptor, positive human epidermal growth factor receptor type 2, and high Ki67 expression. Cancer Sci 100: 1062-1068, 2009.

28 Huang EH, Hynes MJ, Zhang T, Ginestier C, Dontu G, Appelman H, Fields JZ, Wicha MS and Boman BM: Aldehyde dehydrogenase 1 is a marker for normal and malignant human colonic stem cells (SC) and tracks SC overpopulation during colon tumorigenesis. Cancer Res 69: 3382-3389, 2009.

29 Liu X, Wang L, Cui W, Yuan X, Lin L, Cao Q, Wang N, Li Y, Guo W, Zhang X, Wu C and Yang J: Targeting ALDH1A1 by disulfiram/copper complex inhibits non-small cell lung cancer recurrence driven by ALDH-positive cancer stem cells. Oncotarget 7: 58516-58530, 2016.

30 Bellomo C, Caja L and Moustakas A: Transforming growth factor beta as regulator of cancer stemness and metastasis. Br J Cancer 115: 761-769, 2016.

31 Sun M, Zhang N, Wang X, Li Y, Qi W, Zhang H, Li Z and Yang Q: Hedgehog pathway is involved in nitidine chloride induced inhibition of epithelial-mesenchymal transition and cancer stem cells-like properties in breast cancer cells. Cell Biosci 6: 44, 2016.

32 Bao B, Wang Z, Ali S, Kong D, Li Y, Ahmad A, Banerjee S, Azmi AS, Miele L and Sarkar FH: Notch-1 induces epithelialmesenchymal transition consistent with cancer stem cell phenotype in pancreatic cancer cells. Cancer Lett 307: 26-36, 2011.

33 Dembinski JL and Krauss S: Characterization and functional analysis of a slow cycling stem cell-like subpopulation in pancreas adenocarcinoma. Clin Exp Metastasis 26: 611-623, 2009.

34 Marechal R, Bachet JB, Mackey JR, Dalban C, Demetter P, Graham K, Couvelard A, Svrcek M, Bardier-Dupas A, Hammel P, Sauvanet A, Louvet C, Paye F, Rougier P, Penna C, Andre T, Dumontet C, Cass CE, Jordheim LP, Matera EL, Closset J, Salmon I, Deviere J, Emile JF and Van Laethem JL: Levels of gemcitabine transport and metabolism proteins predict survival times of patients treated with gemcitabine for pancreatic adenocarcinoma. Gastroenterology 143: 664-674.e6, 2012.

35 Spratlin J, Sangha R, Glubrecht D, Dabbagh L, Young JD, Dumontet C, Cass C, Lai R and Mackey JR: The absence of human equilibrative nucleoside transporter 1 is associated with reduced survival in patients with gemcitabine-treated pancreas adenocarcinoma. Clin Cancer Res 10: 6956-6961, 2004.

36 Gong W, Zhang X, Wu J, Chen L, Li L, Sun J, Lv Y, Wei X, Du $\mathrm{Y}$, Jin $\mathrm{H}$ and Dong J: RRM1 expression and clinical outcome of gemcitabine-containing chemotherapy for advanced non-smallcell lung cancer: A meta-analysis. Lung Cancer 75: 374-380, 2012. 